\title{
miR-33a Mediates the Anti-Tumor Effect of Lovastatin in Osteosarcoma by Targeting CYR61
}

\author{
Yazeng Huang ${ }^{a, b}$ Jun Zhanga,b Haiyu Shao Jianwen Liu ${ }^{a, b}$ Mengran Jin ${ }^{a, b}$ \\ Jinping Chen ${ }^{\mathrm{a}, \mathrm{b}}$ Hongying Zhao $\mathrm{C}^{\mathrm{b}, \mathrm{c}}$ \\ a'Department of Orthopedics, Zhejiang Provincial People's Hospital, Hangzhou, Zhejiang, 'People's \\ Hospital of Hangzhou Medical College, Hangzhou, Zhejiang, 'Department of Pharmacy, Zhejiang \\ Provincial People's Hospital, Hangzhou, Zhejiang, China
}

\section{Key Words}

Osteosarcoma • Lovastatin - CYR61 - SREBP-2 - miR-33a - Cell invasion • Epithelial-tomesenchymal transition

\begin{abstract}
Background/Aims: Preventing cell metastasis is an effective therapeutic strategy to treat osteosarcoma and improve prognosis. Statins have been found to have anticancer effects in addition to their cholesterol-lowering action. As a new target of statins, cysteine-rich 61 (CYR61) was recently identified to promote cell migration and metastasis in osteosarcoma. However, the underlying mechanisms mediating the regulation of CYR61 expression by statins remain unknown. Methods: Human osteosarcoma cell lines MG63 and SaOS2 were used to clarify the effect of lovastatin on CYR61 expression. Real-time PCR was performed to detect mRNA or microRNA (miRNA) levels and western blot was performed to detect protein levels. Cell invasive ability was determined using Transwell assays. Lentivirus encoding CYR61 CDNA or sterol regulatory element-binding protein 2 (SREBP-2) shRNA was used to upregulate CYR61 expression or downregulate SREBP-2 expression. Binding of the CYR61 3' untranslated region (UTR) and miR-33a was analyzed by luciferase reporter assay. Results: We found that lovastatin treatment decreased CYR61 expression, inhibited cell invasion and altered epithelial-to-mesenchymal-transition (EMT)-related protein expression, while CYR61 overexpression abolished the effect of lovastatin. Moreover, lovastatin increased the expression of SREBP-2 and miR-33a, which were then downregulated by SREBP-2 silencing. Bioinformatics analysis indicated that the CYR61 3'UTR harbored a potential miR-33a binding site and luciferase reporter assay demonstrated that CYR61 was a target of miR-33a in osteosarcoma cells. Furthermore, miR-33a could inhibit cell invasion and alter EMT-related protein expression. SREBP-2 silencing or miR-33a inhibitor upregulated CYR61 expression
\end{abstract}

Y. Huang and J. Zhang contributed equally to this work.

\begin{tabular}{ll}
\hline Hongying Zhao & People's Hospital of Hangzhou Medical College \\
& No. 158, Shangtang Road, Hangzhou, Zhejiang, 310014 (China) \\
& E-Mail drhongyingzhao@126.com
\end{tabular}

\section{KARGER}


and reversed the effects of lovastatin on cell invasion and EMT-related proteins. Conclusion: Our findings suggest lovastatin suppresses osteosarcoma cell invasion through the SREBP-2/ miR-33a/CYR61 pathway.

\section{Introduction}

Osteosarcoma is the most common primary tumor of bone in young people and is characterized by a highly malignant and metastatic potential. Despite recent advancements in the diagnosis and treatment of osteosarcoma, the prognosis and 5-year survival rate of these patients remains poor due to its recurrence and metastasis [1].

Cysteine-rich 61 (CYR61) is a member of the cyr61/CTGF/Nov family [2], whose proteins are associated with the extracellular matrix and cell surface [3]. CYR61 has been reported to mediate cell adhesion, proliferation, and differentiation and has been linked to tumor development [3-5]. CYR61 was found to be overexpressed in breast cancer [6], glioma [7] and pancreatic cancer [8], and downregulated in prostate cancer [9], rhabdomyosarcoma [10] and non-small-cell lung carcinoma [11], suggesting it may play different roles depending on the type of cancer. Recent studies have shown that aberrant expression of CYR61 is associated with osteosarcoma aggressiveness and metastatic potential [12]. CYR61 downregulation reduces osteosarcoma cell metastatic activity and recombinant CYR61 promotes the epithelial-to-mesenchymal transition (EMT) process and metastasis in osteosarcoma $[13,14]$. Interestingly, CYR61 has also been identified as a target of statins and its expression was suppressed by statins in osteosarcoma cells [13]. As inhibitors of 3-hydroxy-3-methylglutaryl-coenzyme A (HMG-CoA) reductase, statins block the conversion of HMG-CoA into mevalonate and are widely used to lower cholesterol. Recently, statins have also been suggested to have anticancer effects by regulating multiple signaling pathways [1517]. However, the underlying mechanisms mediating statin regulation of CYR61 expression remain unknown.

Sterol regulatory element-binding proteins (SREBPs, including SREBP-1 and -2) are a family of basic helix-loop-helix leucine zipper transcription factors that regulate cholesterol and fatty acid synthesis [18]. They are membrane-bound proteins and must undergo proteolytic cleavage to generate active nuclear forms [18]. Specifically, SREBP-2 is involved in cholesterol synthesis and is regulated by intracellular cholesterol levels [19]. Low cholesterol levels likely caused by statins have been thought to activate SREBP-2[20]. The human SREBP-2 gene is located on chromosome 22 and also encodes a microRNA (miRNA) within its intron, miR-33a. MiRNAs are small, non-protein-coding RNAs which act as key mediators in cancer by binding to specific regions in the $3^{\prime}$ untranslated region (UTR) of target mRNA and inhibiting translation or promoting mRNA degradation. MiR-33a is cotranscribed with SREBP-2 and is induced by statins [21]. In addition to controlling critical aspects of cholesterol homeostasis [21], miR-33a was recently shown to function as a tumor suppressor [22-26]. However, whether miR-33a is involved in the regulation of CYR61 expression by statins remains unclear.

Here, we used lovastatin to explore the mechanism mediating the anticancer effect of statins in osteosarcoma. Our data identified CYR61 as a new target gene of miR-33a in osteosarcoma and connected the SREBP-2/miR-33a/CYR61 pathway to lovastatin suppression of osteosarcoma cell invasion.

\section{Materials and Methods}

\section{Cell culture}

Human osteosarcoma cell lines (MG63 and SaOS2) were obtained from the Cell Bank of the Chinese Academy of Sciences (Shanghai, China). Cells were cultured [23] with 10\% fetal calf serum (FCS, Invitrogen, Waltham, MA, USA) at $37^{\circ} \mathrm{C}$ with $5 \% \mathrm{CO}_{2}$. Lovastatin (Sigma-Aldrich, St. Louis, MO, USA) was dissolved in DMSO and used to treat the cells. 


\section{Cellular Physiology Cell Physiol Biochem 2018:51:938-948

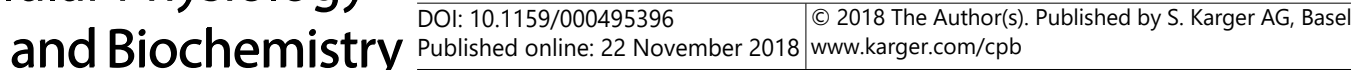

Huang et al.: miR-33a Mediates the Anti-Tumor Effect of Lovastatin

Real-time PCR

Total RNA from cells was extracted with Trizol reagent (Invitrogen) and converted into cDNA using the High-Capacity cDNA Reverse Transcription Kit (Thermo Fisher Scientific Inc., Waltham, MA, USA) according to the manufacturer's instructions. Real-time PCR was performed to detect mRNA and miRNA expression using a PowerUp SYBR Green Master Mix (Thermo Fisher Scientific Inc.) and miRNA Q-PCR Detection Kit (GeneCopoeia, Rockville, MD, USA), respectively. The primers used were as follows: CYR61, 5'-CTCGCCTTAGTCGTCACCC-3' (forward) and 5'-CGCCGAAGTTGCATTCCAG-3' (reverse); SREBP-2, 5'-CCTGGGAGACATCGACGAGAT-3' (forward) and 5'-TGAATGACCGTTGCACTGAAG-3' (reverse); $\beta$-actin, 5'-CACTCTTCCAGCCTTCCTTC-3' (forward) and 5'-GTACAGGTCTTTGCGGATGT-3' (reverse); and U6, 5'-GCTTCGGCAGCACATATACTAAAAT-3' (forward) and 5'-CGCTTCACGAATTTGCGTGTCAT-3' (reverse). $\beta$-actin or U6 gene was used as an internal control for mRNA or miRNA, respectively, and relative amounts of RNA were calculated by the $2^{-\Delta \Delta C t}$ method.

\section{Western blot}

Cells were lysed in cold RIPA lysis buffer (Beyotime, Shanghai, China) and proteins in the supernatants were quantified. After separation by $10 \%$ SDS-PAGE, proteins were transferred onto polyvinylidene difluoride membranes (Life Technologies, Carlsbad, CA, USA). The blots were blocked with 10\% nonfat milk in PBS and then probed with anti-CYR61 (Abcam, Cambridge, MA, USA), anti-SREBP-2 (Santa Cruz, Dallas, TX, USA), anti-E-cadherin, anti-N-cadherin or anti- $\beta$-actin antibody (all from Cell Signaling Technology, Inc., Danvers, MA, USA) for $1 \mathrm{~h}$ at room temperature. After washes, the blots were incubated with HRP-linked secondary antibodies and observed using an enhanced chemiluminescence kit (Pierce Chemical, Rockford, IL, USA). Protein levels were normalized to $\beta$-actin.

\section{Transwell assay}

Transwell assays were performed to determine cell invasive ability using BD Falcon cell culture inserts (BD Biosciences, San Jose, CA, USA) coated with Matrigel (BD Biosciences). After different pretreatments, approximately $1 \times 10^{4}$ cells in $200 \mu \mathrm{L}$ serum-free medium were seeded in the upper chamber, and $800 \mu \mathrm{l}$ of cell culture medium with $10 \% \mathrm{FCS}$ were placed in the lower chamber. After incubation at $37^{\circ} \mathrm{C}$ in $5 \% \mathrm{CO}_{2}$ for $24 \mathrm{~h}$, cells in the upper compartment were removed with a cotton bud and those in the lower compartment were fixed in $10 \%$ formalin and stained with $0.05 \%$ crystal violet. The invading cells were counted using a microscope in four randomly selected fields.

\section{Cell transfection}

The recombinant lentivirus encoding the full-length CYR61 gene was constructed by Hanbio (Shanghai, China) using pHBLV-CMVIE-IRES-Puro lentiviral vectors. The lentivirus encoding SREBP-2 shRNA was purchased from OriGene (Rockville, MD, USA). The empty pHBLV-CMVIE-IRES-Puro lentiviral vectors (Hanbio) and Lenti-shRNA Control Particles (OriGene) were used as negative controls. For stable overexpression of CYR61 or knockdown of SREBP-2, MG63 and SaOS2 cells were infected with the corresponding lentivirus for $36 \mathrm{~h}$ and stable clones selected by puromycin $(10 \mu \mathrm{g} / \mathrm{mL})$ for 2 weeks.

For upregulation and downregulation of miR-33a, $25 \mathrm{nM}$ miR-33a mimics and an miR-33a inhibitor (Ambion, Austin, TX, USA), respectively, were used to transfect MG63 and SaOS2 cells with Lipofectamine 2000 (Invitrogen) following the manufacturer's instructions.

\section{Cell viability assay}

MTT assays were used to examine cell viability. MG63 and SaOS2 cells (10000 cells/per well) treated with or without $10 \mu \mathrm{M}$ lovastatin were plated in a 96-well plate and incubated at $37^{\circ} \mathrm{C}$ in $5 \% \mathrm{CO}_{2}$ for $24 \mathrm{~h}$. MTT solution ( $20 \mu \mathrm{L}$ of $5 \mathrm{mg} / \mathrm{ml}$; Sigma-Aldrich) was then added to each well for $4 \mathrm{~h}$. The supernatant was removed and $100 \mu \mathrm{L}$ of DMSO were added. Absorbance was detected at $570 \mathrm{~nm}$ with a Microplate Reader (Bio-Rad, Hercules, CA, USA).

\section{Luciferase reporter assay}

The CYR61 3'UTR was amplified from MG63 cells and cloned into pmirGLO Vector (Promega, Madison, WI, USA) as pmir-wt-CYR61. Mutations were introduced at potential miR-33a binding sites to construct pmir-mut-CYR61 using the QuikChange Site-Directed Mutagenesis Kit (Stratagene, La Jolla, CA, USA). MG63 


\section{Cellular Physiology Cell Physiol Biochem 2018;51:938-948

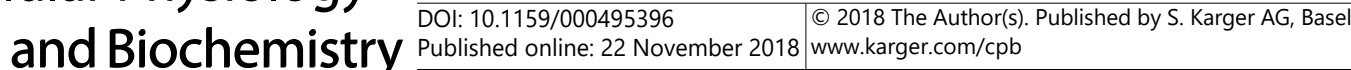 \\ Huang et al.: miR-33a Mediates the Anti-Tumor Effect of Lovastatin}

cells were co-transfected with the pmir-wt-CYR61 vector and miR-33a mimics or mimic-negative control (NC), or with the pmir-mut-CYR61 vector and miR-33a mimics or NC. After transfection for $48 \mathrm{~h}$, luciferase activities were determined using the Dual-Luciferase Reporter Assay System (Promega) according to the manufacturer's instructions.

\section{Statistical analysis}

Data are presented as means \pm standard deviation (SD). Differences between two samples were analyzed for significance using Student's $t$-test. Statistical comparisons of more than two groups were performed using one-way analysis of variance with Bonferroni's post hoc test. $P$ values less than 0.05 were considered statistically significant.

\section{Results}

CYR61 mediates the effect of lovastatin on osteosarcoma cell invasion and EMT-related protein expression

To clarify the effect of lovastatin on the expression of CYR61 in osteosarcoma cells, realtime PCR and western blots were performed after lovastatin treatment. As shown in Fig. 1A, 1-10 $\mu \mathrm{M}$ lovastatin treatment markedly decreased CYR61 mRNA expression and protein levels in MG63 and SaOS2 cells in a dose-dependent manner. To validate the role of CYR61 on the antitumor effect of lovastatin, lentivirus with CYR61 encoding sequence (Lv-CYR61) was constructed and transduced into MG63 and SaOS2 cells (Fig. 1B). After lovastatin treatment, CYR61 mRNA and protein levels were significantly higher in Lv-CYR61-transduced cells than in control cells without transduction (Fig. 1C and D). We then evaluated the effect of lovastatin on cell invasion and EMT-related protein expression and the role of CYR61 in the function of lovastatin. As shown in Fig. 1E, lovastatin treatment reduced cell invasive capability in a dose-dependent manner, while CYR61 overexpression abolished the effect of lovastatin and increased cell invasiveness. Cell viability was also examined (Fig. 1F) to exclude the effect of lovastatin on cell motility [27]. Lovastatin treatment also induced the expression of epithelial marker E-cadherin, but repressed mesenchymal marker N-cadherin, and these effects were reversed by Lv-CYR61 (Fig. 1G).

Lovastatin upregulates miR-33a levels by inducing SREBP-2 expression

To explore the mechanism by which lovastatin induces CYR61 expression, we first determined whether lovastatin affected the expression of SREBP-2 and miR-33a. As shown in Fig. 2A and B, lovastatin increased the expression of SREBP-2 and miR-33a in a dosedependent manner. Western blot assay showed that lovastatin also increased the mature form ( 50-68 kDa) of SREBP-2 (Fig. 2C). Furthermore, we suppressed SREBP-2 expression using lentivirus-mediated RNA interference. The results showed that SREBP-2 silencing significantly decreased SREBP-2 mRNA levels as well as protein levels of the precursor and mature forms of SREBP-2 with or without lovastatin treatment (Fig. 2D-G). MiR-33a expression was also downregulated by SREBP-2 silencing (Fig. 2F), suggesting that lovastatin upregulates miR-33a levels by inducing SREBP-2 expression.

CYR61 is a target gene of miR-33a in osteosarcoma

Bioinformatics analysis indicated that the CYR61 3'UTR harbored a potential miR-33a binding site, implying CYR61 is a target gene of miR-33a. To clarify this, miR-33a mimics were used to increase the miR-33a level (Fig. 3A), and a luciferase reporter vector with a wild-type or mutant CYR61 3'UTR was generated to test the interaction between miR-33a and CYR61 (Fig. 3B). The results of luciferase reporter assays showed that miR-33a mimics significantly decreased luciferase activity in cells transfected with the wild-type CYR61 3'UTR vector, but not in cells transfected with the mutant CYR61 3'UTR vector (Fig. 3C). Moreover, miR-33a led to significantly reduced CYR61 mRNA and protein expression compared with the control (Fig. 3D and E). These results demonstrate that CYR61 is a target of miR-33a in osteosarcoma cells. 
Fig. 1. CYR61 mediates the effect of lovastatin on osteosarcoma cell invasion and EMT. A. MG63 and SaOS2 cells were treated with increasing concentrations of lovastatin for $24 \mathrm{~h}$. CYR61 expression levels were detected by real-time PCR (top) and western blotting (bottom). B. MG63 and SaOS2 cells were stably transfected with Lv-CYR61 or negative control lentivirus (Lv-NC) and CYR61 expression levels were detected by real-time PCR (left) and western blotting (right). MG63 and SaOS2 cells stably transfected with LvCYR61 or negative control lentivirus (Lv-NC) were treated with $10 \mu \mathrm{M}$ lovastatin. CYR61 expression levels were detected by real-time PCR (C) and western blotting (D). E. Cell invasion was measured using Transwell assays. F. MG63 and SaOS2 cells were treated with 10 $\mu \mathrm{M}$ lovastatin for $24 \mathrm{~h}$ and cell viability was detected by MTT assay. G. The expression levels of E-cadherin and $\mathrm{N}$-cadherin were detected using western blotting. All values are presented as means \pm SD from at least three independent experiments. $\quad * \mathrm{p}<0.05$ compared with control; $\# \mathrm{p}<0.05 \quad$ compared with lovastatin-treated cells.

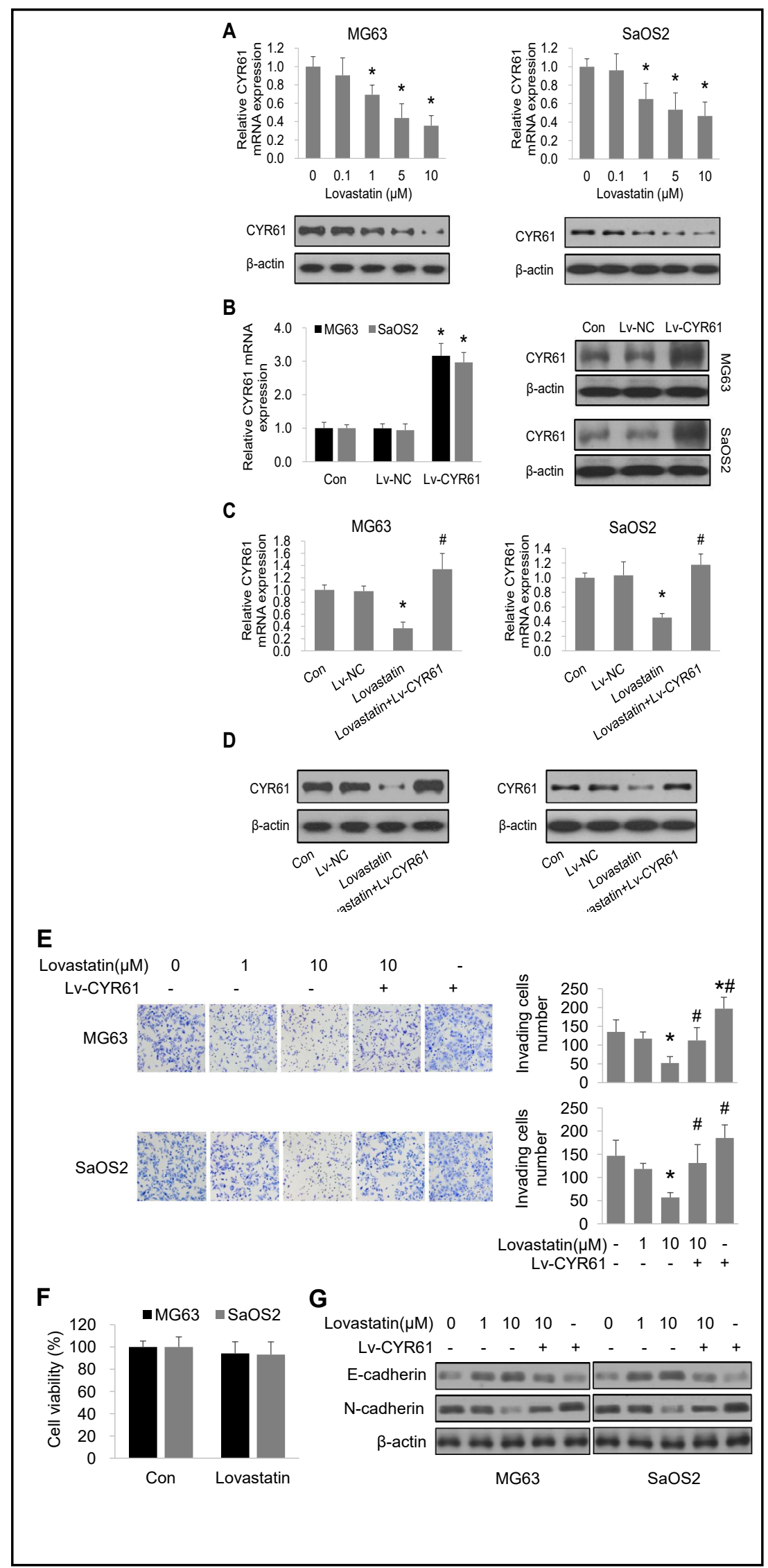




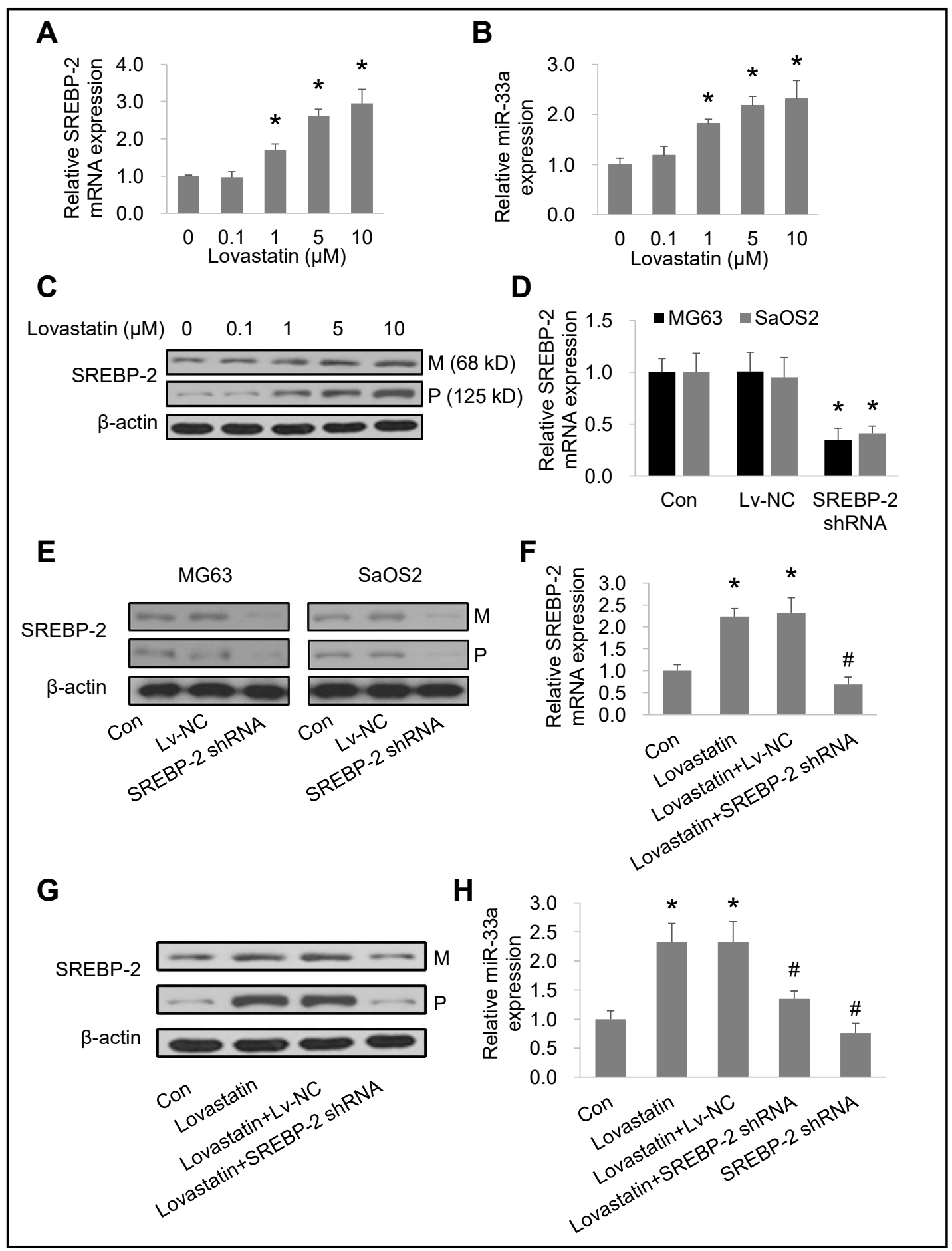

Fig. 2. Lovastatin upregulates miR-33a expression by activating SREBP-2. A-B. MG63 cells were treated with lovastatin at increasing concentrations for $24 \mathrm{~h}$. SREBP-2 (A) and miR-33a (B) expression levels were detected by real-time PCR. C. SREBP-2 protein levels were detected by western blotting. M: mature form; P: precursor form. D-E. MG63 and SaOS2 cells were stably transfected with Lv-SREBP-2 shRNA (SREBP-2 shRNA) or Lv-NC. SREBP-2 expression levels were detected by real-time PCR (D) and western blotting (E). F-G. MG63 cells stably transfected with SREBP-2 shRNA or Lv-NC were treated with $10 \mu$ M lovastatin. SREBP-2 expression levels were detected by real-time PCR (F) and western blotting (G). H. MiR-33a expression levels were detected by real-time PCR. All values are presented as means \pm SD from at least three independent experiments. ${ }^{*} \mathrm{p}<0.05$ compared with control; $\# \mathrm{p}<0.05$ compared with lovastatin-treated cells. 
Fig. 3. CYR61 is a target gene of miR33a in osteosarcoma. A. Real-time PCR was performed to determine the expression level of miR-33a in MG63 cells transfected with scrambled miRNA (miR-NC) or miR-33a mimics (miR-33a). B. The predicted miR-33a binding sites within the wild-type (WT) CYR61 3'-UTR and a mutant type (MT) lacking complementarity with miR-33a are indicated. C. Luciferase reporter vector with WT or MT CYR61 3'-UTR was cotransfected with miR-33a or miR-NC. Luciferase

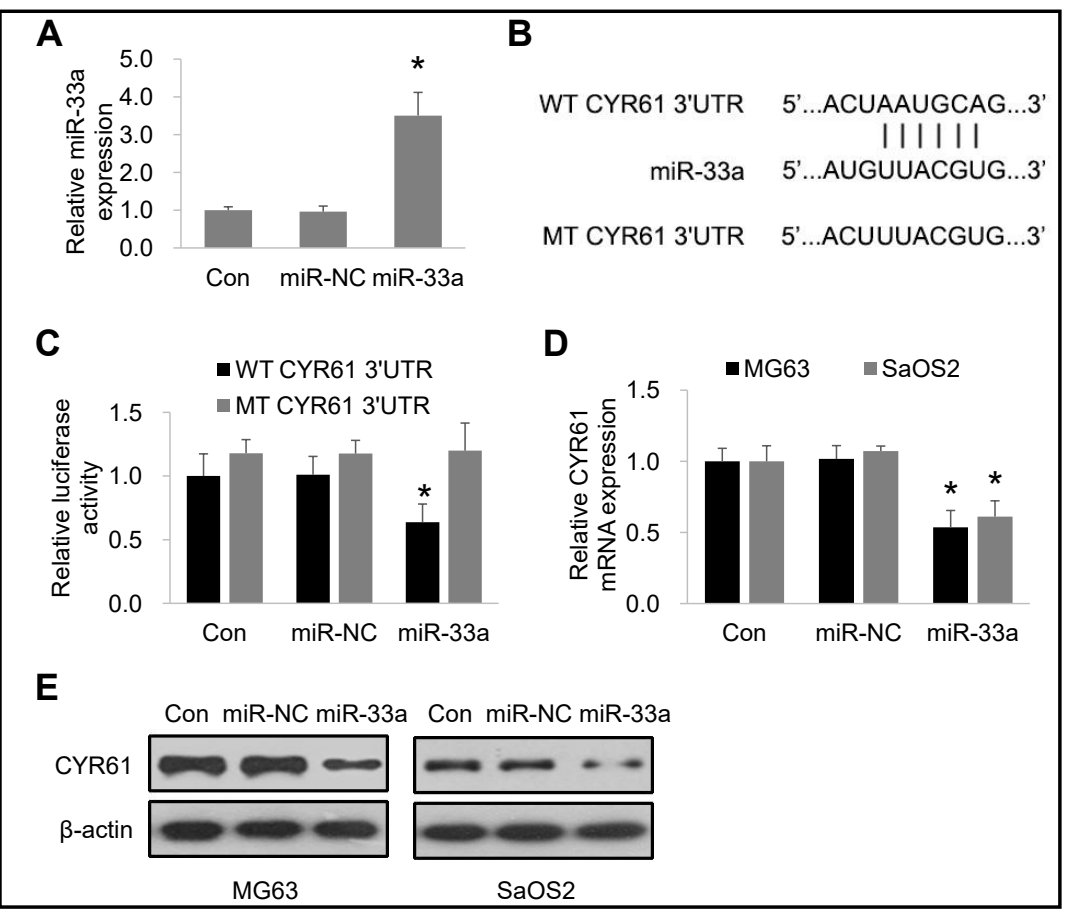
activity was determined after $48 \mathrm{~h}$. D-E. CYR61 expression levels in MG63 cells were detected by real-time PCR (D) and western blotting (E) after transfection with miR-33a or miR-NC. All values are presented as means \pm SD from at least three independent experiments. ${ }^{*} \mathrm{p}<0.05$ compared with control.

Lovastatin suppresses osteosarcoma cell invasion through the SREBP-2/miR-33a/CYR61 pathway

We further verified whether the regulatory effect of lovastatin on CYR61 expression was through the SREBP-2/miR-33a pathway. As shown in Fig. 4A and B, SREBP-2 silencing and miR-33a inhibition significantly upregulated CYR61 expression in cells treated with lovastatin. To clarify whether the SREBP-2/miR-33a/CYR61 pathway was involved in the suppression of osteosarcoma cell invasion and EMT by lovastatin, MG63 and SaOS2 cells were transfected with miR-33a mimics, an miR-33a inhibitor, Lv-SREBP-2 shRNA or were co-transfected with Lv-CYR61 and miR-33a mimics. As shown in Fig. 4C and D and similar to lovastatin treatment, miR-33a inhibited cell invasion and altered EMT-related protein expression, while SREBP-2 silencing or miR-33a inhibition by an miR-33a inhibitor significantly increased the number of invading cells and $\mathrm{N}$-cadherin expression and repressed E-cadherin expression compared with the lovastatin group. CYR61 overexpression reversed the effects of miR-33a on cell invasion and EMT-related proteins. These results suggest that lovastatin suppresses osteosarcoma cell invasion and alters EMT-related protein expression through the SREBP-2/miR-33a/CYR61 pathway. 


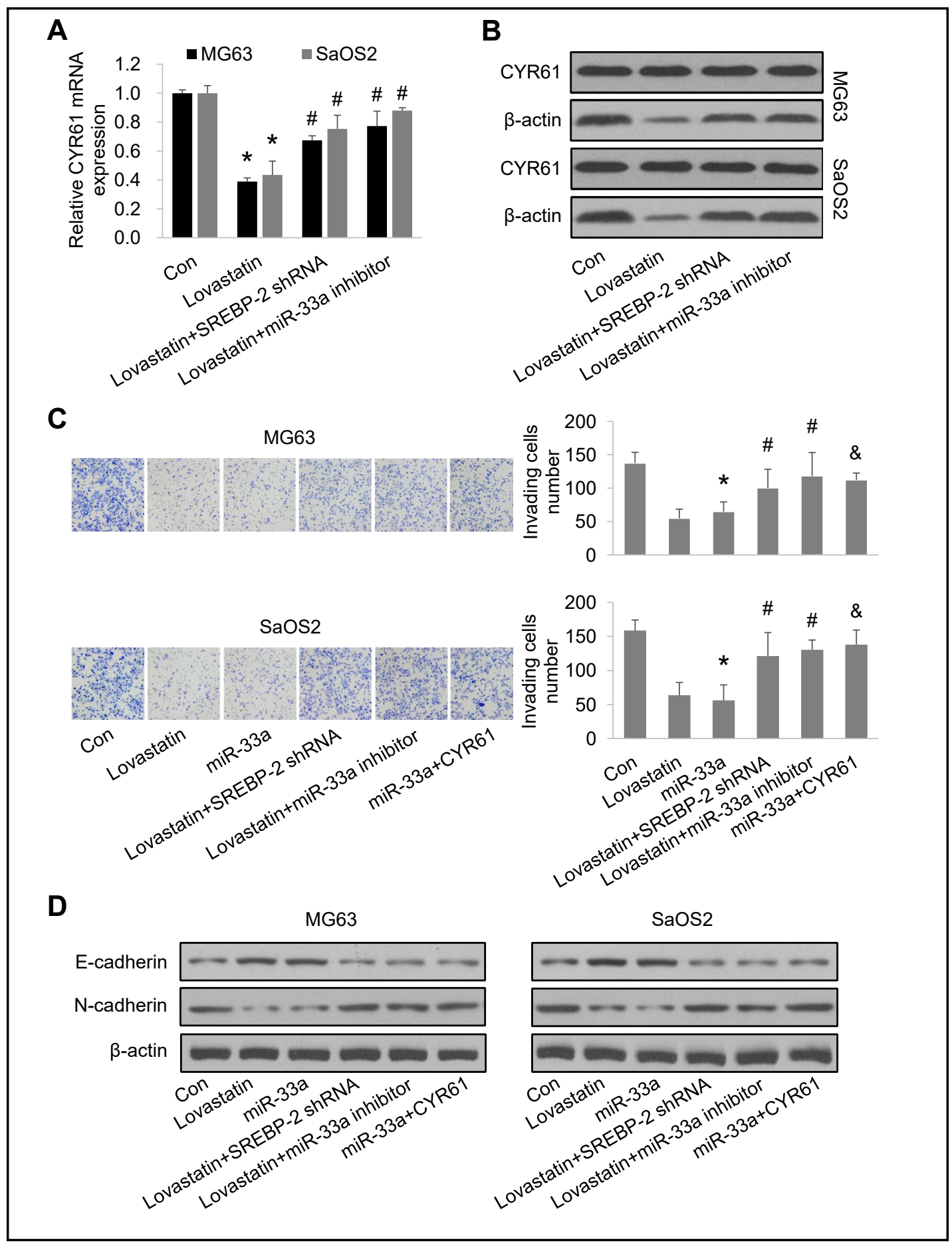

Fig. 4. Lovastatin suppresses osteosarcoma cell invasion and EMT through the SREBP-2/miR-33a/CYR61 pathway. A-B. MG63 and SaOS2 cells were transfected with SREBP-2 shRNA or miR-33a inhibitor and then treated with $10 \mu \mathrm{M}$ lovastatin for $24 \mathrm{~h}$. CYR61 expression levels were detected by real-time PCR (A) and western blotting (B). C. MG63 and SaOS2 cells were transfected with miR-33a, Lv-SREBP-2 shRNA or an miR-33a inhibitor and co-transfected with Lv-CYR61 and miR-33a before treatment with lovastatin. Cell invasion was measured using Transwell assays. D. Expression levels of E-cadherin and N-cadherin were detected using western blotting. All values are presented as means \pm SD from at least three independent experiments. ${ }^{*} \mathrm{p}<0.05$ compared with control; $\# \mathrm{p}<0.05$ compared with the lovastatin-treated cells; $\& \mathrm{p}<0.05$ compared with miR-33a-transfected cells.

\section{KARGER}




\section{Cellular Physiology Cell Physiol Biochem 2018;51:938-948 and Biochemistry Published \begin{tabular}{l|l} 
DOI: 10.1159/000495396 & 2018 The Author(s). Published by S. Karger AG, Basel \\
www.karger.com/cpb
\end{tabular} \\ Huang et al.: miR-33a Mediates the Anti-Tumor Effect of Lovastatin}

\section{Discussion}

In this study, we showed that lovastatin induced the co-expression of SREBP-2 and miR33a in osteosarcoma cells. MiR-33a inhibited cell invasion and altered EMT-related protein expression by targeting CYR61. These results suggest a potential mechanism underlying the anticancer effect of statins.

In addition to their wide use as cholesterol-lowering agents, statins are currently under consideration as potential drugs for cancer therapy [28]. Anticancer effects of statins have been reported in a variety of cancer types including osteosarcoma [29-31]. Although RhoAmediated signaling has been shown to be involved in simvastatin-induced apoptosis and atorvastatin-reduced invasion of osteosarcoma cells [17, 32], Olivia et al. identified CYR61 as a new target of atorvastatin that was independent from RhoA signaling [13]. CYR61 has been reported to be an oncogene and is associated with osteosarcoma aggressiveness and metastatic potential [11].

Lovastatin is the first statin to be approved by the US FDA as a cholesterol-lowering drug, but its effects on osteosarcoma development have yet to be thoroughly evaluated. Here, we showed that lovastatin treatment markedly decreased CYR61 expression levels and that CYR61 overexpression abolished the effect of lovastatin on cell invasion and EMTrelated proteins, suggesting that CYR61 mediates the anticancer effects of lovastatin on osteosarcoma cells.

MiR-33a is located within an intron of the SREBP2 gene and has been found to mediate the hepatotoxic effects of statins. Bioinformatics analysis showed that CYR61 is a potential target of miR-33a. Therefore, we proceeded to investigate whether miR-33a mediates the regulation of lovastatin on CYR61. As the host gene of miR-33a, SREBP-2 is activated in response to the reduction in hepatic cholesterol content as well as by statins [19]. Activated SREBP-2 in its mature form then enters the nucleus and activates transcription of genes involved in cholesterol homeostasis [18]. Our study showed that lovastatin not only increased the mature form of SREBP-2, but also increased total SREBP-2 expression. Considering that sterol regulatory elements are present in its promoter [33], SREBP-2 may induce its own expression in a manner similar to SREBP-1[34]. This is also consistent with results showing that miR-33a expression was downregulated by SREBP-2 shRNA.

MiR-33a was recently identified as a tumor suppressor in melanoma, non-small cell lung cancer, and breast cancer by targeting multiple genes [22-24]. Here, we found that miR-33a could regulate CYR61 by binding to its 3'-UTR in osteosarcoma cells. CYR61 plays an important role in osteosarcoma invasion, EMT, and metastasis via a signal transduction pathway that involves $\alpha v \beta 5$ integrin, Raf-1, MEK, ERK, and Elk-1[13, 14]. The suppressive effect of miR-33a on osteosarcoma cell invasion and EMT-related protein expression, similar to the effect of lovastatin, was also mediated by CYR61. SREBP-2 silencing or an miR-33a inhibitor significantly attenuated the effects of lovastatin on cell invasion and EMT-related proteins, suggesting that the SREBP-2/miR-33a/CYR61 pathway mediated the anticancer effect of statins in osteosarcoma. Interestingly, CYR61 was also found to activate the SREBP pathway and upregulate fatty acid synthase protein expression in breast cancer cells [35]. There may be a feedback loop between SREBP-2 and CYR61 or crosstalk with fatty acid metabolism that is worth further investigation.

\section{Conclusion}

Our data demonstrate that lovastatin regulates CYR61 expression by activating SREBP-2 and inducing miR-33a expression. This pathway mediates the suppressive effects of lovastatin on osteosarcoma cell invasion. This study provides a molecular basis for the use of statins in cancer therapy and suggests potential targets for osteosarcoma treatment. 


\section{Cellular Physiology Cell Physiol Biochem 2018;51:938-948 and Biochemistry \begin{tabular}{l|l} 
DOI: 10.1159/000495396 & (c)18 The Author(s). Published by S. Karger AG, Basel \\
Publishw.karger.com/cpb
\end{tabular}

\section{Acknowledgements}

This study was supported by grants from the Zhejiang Provincial Natural Science Foundation of China (LY14H060006), (LY15H060006) and (LY16H060007), Natural Scientific Research Foundation of Zhejiang Medical College (2011XZB01), Zhejiang Provincial Health Bureau Science Foundation of China (2012KYB022) and Zhejiang Provincial Chinese Traditional Medicine Bureau Science Foundation of China (2012ZB015).

\section{Disclosure Statement}

The authors disclose no competing interests in this research.

\section{References}

1 Thompson LD: Osteosarcoma. Ear Nose Throat J 2013;92:288, 290.

-2 Lau LF: Cell surface receptors for CCN proteins. J Cell Commun Signal 2016;10:121-127.

3 Yang GP, Lau LF: Cyr61, product of a growth factor-inducible immediate early gene, is associated with the extracellular matrix and the cell surface. Cell Growth Differ 1991;2:351-357.

4 Babic AM, Kireeva ML, Kolesnikova TV, Lau LF: CYR61, a product of a growth factor-inducible immediate early gene, promotes angiogenesis and tumor growth. Proc Natl Acad Sci U S A 1998;95:6355-6360.

-5 Huang X, Xiang L, Li Y, Zhao Y, Zhu H, Xiao Y, Liu M, Wu X, Wang Z, Jiang P, Qing H, Zhang Q, Liu G, Zhang W, Li A, Chen Y, Liu S, Wang J: Snail/FOXK1/Cyr61 Signaling Axis Regulates the Epithelial-Mesenchymal Transition and Metastasis in Colorectal Cancer.

-6 Xie D, Nakachi K, Wang H, Elashoff R, Koeffler HP: Elevated levels of connective tissue growth factor, WISP-1, and CYR61 in primary breast cancers associated with more advanced features. Cancer Res 2001;61:8917-8923.

7 Xie D, Yin D, Wang HJ, Liu GT, Elashoff R, Black K, Koeffler HP: Levels of expression of CYR61 and CTGF are prognostic for tumor progression and survival of individuals with gliomas. Clin Cancer Res 2004;10:20722081.

-8 Holloway SE, Beck AW, Girard L, Jaber MR, Barnett CC, Jr., Brekken RA, Fleming JB: Increased expression of Cyr61 (CCN1) identified in peritoneal metastases from human pancreatic cancer. J Am Coll Surg 2005;200:371-377.

-9 Pilarsky CP, Schmidt U, Eissrich C, Stade J, Froschermaier SE, Haase M, Faller G, Kirchner TW, Wirth MP: Expression of the extracellular matrix signaling molecule Cyr61 is downregulated in prostate cancer. Prostate 1998;36:85-91.

10 Croci S, Landuzzi L, Astolfi A, Nicoletti G, Rosolen A, Sartori F, Follo MY, Oliver N, De Giovanni C, Nanni P, Lollini PL: Inhibition of connective tissue growth factor (CTGF/CCN2) expression decreases the survival and myogenic differentiation of human rhabdomyosarcoma cells. Cancer Res 2004;64:1730-1736.

11 Tong X, Xie D, O'Kelly J, Miller CW, Muller-Tidow C, Koeffler HP: Cyr61, a member of CCN family, is a tumor suppressor in non-small cell lung cancer. J Biol Chem 2001;276:47709-47714.

12 Sabile AA, Arlt MJ, Muff R, Bode B, Langsam B, Bertz J, Jentzsch T, Puskas GJ, Born W, Fuchs B: Cyr61 expression in osteosarcoma indicates poor prognosis and promotes intratibial growth and lung metastasis in mice. J Bone Miner Res 2012;27:58-67.

13 Fromigue O, Hamidouche Z, Vaudin P, Lecanda F, Patino A, Barbry P, Mari B, Marie PJ: CYR61 downregulation reduces osteosarcoma cell invasion, migration, and metastasis. J Bone Miner Res 2011;26:1533-1542.

14 Hou CH, Lin FL, Hou SM, Liu JF: Cyr61 promotes epithelial-mesenchymal transition and tumor metastasis of osteosarcoma by Raf-1/MEK/ERK/Elk-1/TWIST-1 signaling pathway. Mol Cancer 2014;13:236.

15 Jang HJ, Hong EM, Kim M, Kim JH, Jang J, Park SW, Byun HW, Koh DH, Choi MH, Kae SH, Lee J: Simvastatin induces heme oxygenase-1 via NF-E2-related factor 2 (Nrf2) activation through ERK and PI3K/Akt pathway in colon cancer. Oncotarget 2016;7:46219-46229. 


\section{Cellular Physiology Cell Physiol Biochem 2018;51:938-948 and Biochemistry DOl: 10.1159/000495396 2018 O 2018 The Author(s). Published by S. Karger AG, Basel

-16 Jung HH, Lee SH, Kim JY, Ahn JS, Park YH, Im YH: Statins affect ETS1-overexpressing triple-negative breast cancer cells by restoring DUSP4 deficiency. Sci Rep 2016;6:33035.

17 Fromigue 0, Hamidouche Z, Marie PJ: Blockade of the RhoA-JNK-c-Jun-MMP2 cascade by atorvastatin reduces osteosarcoma cell invasion. J Biol Chem 2008;283:30549-30556.

-18 Shimano H: Sterol regulatory element-binding proteins (SREBPs): transcriptional regulators of lipid synthetic genes. Prog Lipid Res 2001;40:439-452.

-19 Miao J, Haas JT, Manthena P, Wang Y, Zhao E, Vaitheesvaran B, Kurland IJ, Biddinger SB: Hepatic insulin receptor deficiency impairs the SREBP-2 response to feeding and statins. J Lipid Res 2014;55:659-667.

20 Scharnagl H, Schinker R, Gierens H, Nauck M, Wieland H, Marz W: Effect of atorvastatin, simvastatin, and lovastatin on the metabolism of cholesterol and triacylglycerides in HepG2 cells. Biochem Pharmacol 2001;62:1545-1555.

-21 Marquart TJ, Allen RM, Ory DS, Baldan A: miR-33 links SREBP-2 induction to repression of sterol transporters. Proc Natl Acad Sci U S A 2010;107:12228-12232.

-22 Zhou J, Xu D, Xie H, Tang J, Liu R, Li J, Wang S, Chen X, Su J, Zhou X, Xia K, He Q, Chen J, Xiong W, Cao P, Cao K: miR-33a functions as a tumor suppressor in melanoma by targeting HIF-1alpha. Cancer Biol Ther 2015;16:846-855.

23 Yang L, Yang J, Li J, Shen X, Le Y, Zhou C, Wang S, Zhang S, Xu D, Gong Z: MircoRNA-33a inhibits epithelial-tomesenchymal transition and metastasis and could be a prognostic marker in non-small cell lung cancer. Sci Rep 2015;5:13677.

-24 Zhang C, Zhang Y, Ding W, Lin Y, Huang Z, Luo Q: MiR-33a suppresses breast cancer cell proliferation and metastasis by targeting ADAM9 and ROS1. Protein Cell 2015;6:881-889.

-25 Zhang M, Gong W, Zuo B, Chu B, Tang Z, Zhang Y, Yang Y, Zhou D, Weng M, Qin Y, Ma M, Jiang A, Ma F, Quan Z: The microRNA miR-33a suppresses IL-6-induced tumor progression by binding Twist in gallbladder cancer. Oncotarget 2016;7:78640-78652.

-26 Du M, Zhang Y, Mao Y, Mou J, Zhao J, Xue Q, Wang D, Huang J, Gao S, Gao Y: MiR-33a suppresses proliferation of NSCLC cells via targeting METTL3 mRNA. Biochem Biophys Res Commun 2017;482:582-589.

-27 Fromigue O, Hay E Fau - Modrowski D, Modrowski D Fau - Bouvet S, Bouvet S Fau - Jacquel A, Jacquel A Fau - Auberger P, Auberger P Fau - Marie PJ, Marie PJ: RhoA GTPase inactivation by statins induces osteosarcoma cell apoptosis by inhibiting p42/p44-MAPKs-Bcl-2 signaling independently of BMP-2 and cell differentiation.

28 Brown AJ: Cholesterol, statins and cancer. Clin Exp Pharmacol Physiol 2007;34:135-141.

-29 Yang T, Yao H, He G, Song L, Liu N, Wang Y, Yang Y, Keller ET, Deng X: Effects of Lovastatin on MDA-MB-231 Breast Cancer Cells: An Antibody Microarray Analysis. J Cancer 2016;7:192-199.

-30 Parikh A, Childress C, Deitrick K, Lin Q, Rukstalis D, Yang W: Statin-induced autophagy by inhibition of geranylgeranyl biosynthesis in prostate cancer PC3 cells. Prostate 2010;70:971-981.

-31 Fromigue 0, Hamidouche Z, Marie PJ: Statin-induced inhibition of 3-hydroxy-3-methyl glutaryl coenzyme a reductase sensitizes human osteosarcoma cells to anticancer drugs. J Pharmacol Exp Ther 2008;325:595600.

-32 Kamel WA, Sugihara E, Nobusue H, Yamaguchi-Iwai S, Onishi N, Maki K, Fukuchi Y, Matsuo K, Muto A, Saya H, Shimizu T: Simvastatin-Induced Apoptosis in Osteosarcoma Cells: A Key Role of RhoA-AMPK/p38 MAPK Signaling in Antitumor Activity. Mol Cancer Ther 2017;16:182-192.

-33 Tao R, Xiong X, DePinho RA, Deng CX, Dong XC: Hepatic SREBP-2 and cholesterol biosynthesis are regulated by FoxO3 and Sirt6. J Lipid Res 2013;54:2745-2753.

-34 Amemiya-Kudo M, Shimano H, Yoshikawa T, Yahagi N, Hasty AH, Okazaki H, Tamura Y, Shionoiri F, Iizuka Y, Ohashi K, Osuga J, Harada K, Gotoda T, Sato R, Kimura S, Ishibashi S, Yamada N: Promoter analysis of the mouse sterol regulatory element-binding protein-1c gene. J Biol Chem 2000;275:31078-31085.

-35 Menendez JA, Vellon L, Espinoza I, Lupu R: The metastasis inducer CCN1 (CYR61) activates the fatty acid synthase (FASN)-driven lipogenic phenotype in breast cancer cells. Oncoscience 2016;3:242-257. 